\title{
Editorial
}

\section{Jaroslaw Drelich}

Houghton, MI, USA

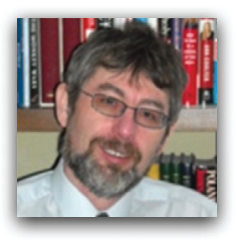

Three volumes of Surface Innovations are already in the record. With this first issue of 2016, we enter into our fourth year of operation. Before my brief review of the content of this issue, I would like to mention some recent and important developments.

It is my great pleasure to inform the readers that Surface Innovations has been accepted into Thomson Reuters Indices SCIE, Current Contents and JCR. Mr. Ben Ramster, Journals Manager, and Dr. Alison McAnena, Journal Development Editor, at ICE Publishing were the driving force behind this initiative and I would like to express my appreciation to them for their efforts. As a result, the papers published in Surface Innovations are already reported in Web of Science. The journal will receive its first impact factor in the annual announcement by Thomson Reuters in June 2016. I hope that our presence in Web of Science will make Surface Innovations more visible and attractive to the international scientific community, intensifying demands for papers and increasing their citation in the months to come.

Citation of papers published in Surface Innovations has risen in recent months as per Google Scholar record. Five of the top mostcited papers of 2015 include papers on the guidelines to contact angle measurements, ${ }^{1}$ the design of superoleophobic surfaces, ${ }^{2}$ the physics behind superhydrophobic and superhydrophilic surfaces, ${ }^{3}$ superhydrophobic coatings of magnesium alloys ${ }^{4}$ and spray deposition of superhydrophobic nanocoatings. ${ }^{5}$ Four out of five of these papers are on manipulation of surface roughness and chemistry of materials to enhance liquid-repelling properties, a subject that still remains one of the most popular research topics worldwide among researchers studying and modifying surfaces. High citation of the first paper on measurements of contact angles reveals a far-reaching demand for papers addressing reproducible measurement methodologies and protocols, often not properly developed and executed by new users of instrumentation who have contributed to the explosion of publications on the measurements of contact angles in recent years. The abuse of terminology and misuse of scientific concepts, such as in many papers on superhydrophobicity, contact angles and wetting phenomena, are spreading throughout the scientific literature at an alarming rate as mentioned in previous review papers ${ }^{3,6}$ and more profoundly addressed recently by Della Volpe and Siboni. ${ }^{7}$ Surface Innovations will continue to provide a platform for articles and notes on proper use of terminology and methodology. New initiatives and contributions into this field are expected as early as in the second half of 2016 or at the beginning of 2017, and will likely involve some of our Editorial Board members. Unsolicited contributions addressing the proper use of surface chemistry terms and measuring methods are welcome, and will receive proper consideration from the Editor.

This issue concludes the two-part special issue of Surface Innovations on recent developments in polymer surface modification and is dedicated to Dr. Kashmiri L. Mittal on the occasion of his 70th birthday in recognition of his impacts on the field of adhesion science and technology. ${ }^{8}$ The issue starts with a technical report on a 'green' fiber surface modification approach to enhance hydrophobicity of cotton fabrics. ${ }^{9}$ Zhong and Netravali describe grafting of aliphatic fatty chains to cotton fiber that converts hydrophilic surface of fibers to a hydrophobic one. Fiber hydrophobicity that results from surface grafting lasts at an acceptable level through 37 cycles of laundry washes. Another contribution by Bahners and Gutmann touches the topic of fiber surface modification, although the primary emphasis is on properties and characteristics of polymeric coatings. ${ }^{10}$ The authors briefly review ultraviolet-induced graft copolymerization and photopolymerization methods used in functionalization of fibers, and then review their broad range of studies on bulk properties, chemical stability and characteristics of polymeric coatings with thickness less than $500 \mathrm{~nm}$.

In the third contribution, Catiker et al. describe patterning of cavities and holes in polymers using $\mathrm{KrF}$ excimer laser, and then use the patterned polymers to study their interactions with human fibroblast cells. ${ }^{11}$ They demonstrate good reproducibility and precision in patterning of cavities and holes with a size of 20-43 microns. More importantly, they show that the topographical patterns can be used to control attachment and growth of human fibroblast cells on polymers. The use of polymers with modified surfaces to study their interactions with biological entities is also discussed by Thakur et al. ${ }^{12}$ The authors demonstrate that hydrophobicity of polyethylene can be reduced with lowpressure nitrogen plasma and the less hydrophobic polyethylene surface demonstrates improved biofouling resistance. In the final contribution to this issue, Abdulal et al. compare chemical functionality of poly(ethylene 2,6-naphthalate) with a surface modified through either ozonation or UV photooxidation methods. ${ }^{13}$ They conclude that the UV photooxidation method produces polymers with more hydrophilic surfaces, a result of achieving a higher oxidation and formation of anhydride/carbonate moieties.

I hope you will find something interesting in this first issue of 2016 and would like to remind you that we always welcome your feedback on the contents of Surface Innovations. 


\section{REFERENCES}

1. Drelich J (2013) Guidelines to measurements of reproducible contact angles using a sessile-drop technique. Surface Innovations 1(4): 248-254.

2. Kota AK, Mabry JM and Tuteja A (2013) Superoleophobic surfaces: design criteria and recent studies. Surface Innovations 1(2): 77-89.

3. Drelich J and Marmur A (2014) Physics and applications of superhydrophobic and superhydrophilic surfaces and coatings. Surface Innovations 2(4): 211-227.

4. Boinovich LB, Emelyanenko AM, Pashinin AS et al. (2013) $\mathrm{Mg}$ alloy treatment for superhydrophobic anticorrosion coating formation. Surface Innovations 1(3): 162-172.

5. Yeong YH, Davis A, Steele A, Loth E and Bayer IS (2014) Spray deposition effects on superhydrophobicity and durability of nanocoatings. Surface Innovations 2(2): 70-78.

6. Drelich J, Chibowski E, Meng DD and Terpilowski K (2011) Hydrophilic and superhydrophilic surfaces and materials. Soft Matter 7(21): 9804-9828.
7. Della Volpe C and Siboni S (2015) Use, abuse, misuse and proper use of contact angles: a critical review. Reviews of Adhesion and Adhesives 3(4): 365-385.

8. Gutowski WS and Drelich J (2015) Dedication. Surface Innovations 3(4): 190-191.

9. Zhong Y and Netravali AN (2016) 'Green' surface treatment for water repellent cotton fabrics. Surface Innovations 4(1): 3-13.

10. Bahners T and Gutmann JS (2016) Using bulk properties of UV-polymerized thin layers for fiber modification. Surface Innovations 4(1): 14-22.

11. Catiker E, Stakleff KS, Carr KB and Sancaktar E (2016) Laser perforated polymer films for possible use in tissue engineering. Surface Innovations 4(1): 23-32.

12. Thakur S, Pal D and Neogi S (2016) Prevention of biofilm attachment by plasma treatment of polyethylene. Surface Innovations 4(1): 33-38.

13. Abdulal EA, Bailey A, Mehan M et al. (2016) Surface modification of PEN treated with ozone and UV photooxidation. Surface Innovations 4(1): 39-47. 